\title{
Résultats en ce qui concerne les participants autochtones sondés dans le cadre de l'enquête Track auprès des utilisateurs de drogues injectables au Canada, phase 4, 2017 à 2019
}

\author{
Jill Tarasuk ${ }^{\star}$, Meghan Sullivan², Donna Bush³, Christian Hui, Melissa Morris, Tami Starlight, \\ François Cholette ${ }^{4}$, Leigh Jonah', Maggie Bryson', Dana Paquette', Renée Masching ${ }^{2}$
}

\section{Résumé}

Contexte : L'enquête Track auprès des utilisateurs de drogues injectables a permis de recueillir des données dans quatorze sites sentinelles au Canada (2017 à 2019). Ces résultats décrivent la prévalence du virus de l'immunodéficience humaine $(\mathrm{VIH})$, de l'hépatite $\mathrm{C}$ et des comportements à risque associés à ceux-ci chez les participants autochtones.

Méthodes : Des informations sur les caractéristiques sociodémographiques, les déterminants sociaux de la santé, le recours aux services de prévention et au dépistage, la consommation de drogues, les comportements à risque, ainsi que le dépistage, les soins et le traitement du VIH et de l'hépatite C ont été recueillies par l'entremise de questionnaires administrés par un intervieweur. Les échantillons biologiques ont été analysés pour y détecter la présence d'anticorps anti-VIH et anti-hépatite $C$ et l'acide ribonucléique (ARN) de l'hépatite $C$. Les statistiques descriptives ont été calculées et examinées par un groupe consultatif dirigé par des autochtones, selon l'approche à double perspective (Two-Eyed Seeing).

Résultats : Parmi les 2383 participants, 997 étaient des autochtones (82,9\% étaient des membres des Premières Nations, $14,9 \%$ étaient des Métis et 2,2 \% étaient des Inuits). Plus de la moitié $(54,5 \%)$ étaient des hommes cisgenres et l'âge moyen était de 38,9 ans. Une grande proportion $(84,0 \%)$ des participants ont déclaré que leur santé mentale était de " passable excellente ». Une forte proportion d'entre eux ont été victimes de stigmatisation et de discrimination $(90,2 \%)$ ainsi que de violences physiques, sexuelles et/ou psychologiques durant l'enfance (87,5\%) ou de la part d'un partenaire sexuel $(78,6 \%)$. Un pourcentage élevé d'entre eux ont déclaré utiliser un programme de distribution de seringues $(90,5 \%)$ et avoir été dépisté pour le $\mathrm{VIH}(87,9 \%)$ et l'hépatite $\mathrm{C}(87,8 \%)$. La prévalence du VIH était de 15,4\% (78,2 \% d'entre eux avaient connaissance de leur statut infectieux) et $36,4 \%$ d'entre eux étaient séropositifs pour l'ARN de l'hépatite $C(49,4 \%$ d'entre eux avaient connaissance de leur statut infectieux).

Conclusion : L'enquête a révélé des taux élevés de VIH et d'hépatite $\mathrm{C}$. Elle a également révélé des défis liés à l'accès et au maintien des soins et des traitements liés au VIH et à l'hépatite $\mathrm{C}$. Ces renseignements éclairent les stratégies de réduction des méfaits, y compris la nécessité d'accroître la sensibilisation à la prophylaxie d'une manière culturellement pertinente.

Citation proposée : Tarasuk J, Sullivan M, Bush D, Hui C, Morris M, Starlight T, Cholette F, Jonah L, Bryson M, Paquette $D$, Masching R. Résultats en ce qui concerne les participants autochtones sondés dans le cadre de I'enquête Track auprès des utilisateurs de drogues injectables au Canada, phase 4, 2017 à 2019. Relevé des maladies transmissibles au Canada 2021;47(1):43-55. https://doi.org/10.14745/ccdr.v47i01a07f

Mots-clés : $\mathrm{VIH}$, hépatite $\mathrm{C}$, autochtones qui s'injectent des drogues, consommation de drogues, comportements d'injection, pratiques sexuelles à risque, surdose, statut d'infection, dépistage, soins et traitement
Cette oeuvre est mise à la disposition selon les termes de la licence internationale Creative Commons Attribution 4.0

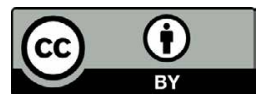

Affiliations

${ }^{1}$ Centre de la lutte contre les maladies transmissibles et les infections, Agence de la santé publique du Canada, Ottawa, ON

2 Réseau canadien autochtone du sida, Halifax, NS

${ }^{3}$ Association des femmes Inuites du Canada (Pauktuutit), Ottawa, ON

${ }^{4}$ Laboratoire national de microbiologie, Agence de la santé publique du Canada, Winnipeg, MB

*Correspondance :

jill.tarasuk@canada.ca 


\section{Introduction}

Au Canada, les peuples autochtones représentent 4,9\% de la population canadienne totale (1); toutefois, ils sont touchés de façon disproportionnée par le virus de l'immunodéficience humaine $(\mathrm{VIH})$ et les infections par le virus de l'hépatite $\mathrm{C}$. En 2016 , on a estimé que $11,3 \%$ des nouvelles infections au VIH au Canada touchaient les peuples autochtones (2) et que les infections à l'hépatite $C$ nouvellement diagnostiquées parmi les membres des Premières Nations vivant dans les réserves étaient trois fois plus élevées que les nouveaux diagnostics dans l'ensemble de la population canadienne (3). La surveillance nationale du VIH fondée sur des cas pour 2017 a révélé que $68,1 \%$ des cas attribués aux utilisateurs de drogues injectables ont déclaré être d'origine autochtone, parmi les $49,3 \%$ cas déclarés de VIH pour lesquels des données sur l'origine ethnique sont disponibles (4).

L'Agence de la santé publique du Canada (l'Agence), en collaboration avec des partenaires provinciaux, territoriaux et locaux en santé publique, surveille les tendances en ce qui concerne la prévalence du VIH et de l'hépatite $\mathrm{C}$ et les facteurs de risque connexes dans les populations clés, comme les utilisateurs de drogues injectables, par l'entremise du système de surveillance Track. L'enquête Track auprès des utilisateurs de drogues injectables (auparavant dénommée "I-Track») consiste en la réalisation d'enquêtes transversales répétées dans certains sites sentinelles du Canada. Elle a été mise en œuvre pour la première fois de 2003 à 2005 (phase 1) dans sept sites sentinelles. Trois périodes de collecte de données ont suivi, notamment la plus récente, la phase 4 (2017 à 2019), dans 14 sites sentinelles.

Des renseignements sur les pratiques à risque et les comportements favorisant la santé chez les populations les plus exposées au risque d'infection au $\mathrm{VIH}$, incluant les utilisateurs de drogues injectables autochtones, sont nécessaires pour mieux comprendre les facteurs contribuant à la transmission (5). Ce rapport vise à présenter les résultats de surveillance nationale réalisée auprès des participants autochtones à la phase 4 de l'enquête Track auprès des utilisateurs de drogues injectables

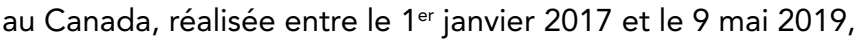
dans les sites sentinelles canadiens participants. Ces résultats fournissent des renseignements sur les caractéristiques sociodémographiques, les déterminants sociaux de la santé, le recours aux services de prévention et au dépistage des infections transmises sexuellement et par le sang, la consommation de drogues et les antécédents de surdose, les comportements sexuels à risque ainsi que la prévalence et la cascade de soins du $\mathrm{VIH}$ et de l'hépatite $\mathrm{C}$ et la connaissance du statut d'infection à ces maladies.

\section{Méthodes}

\section{Source de données et techniques d'échantillonnage}

Les données présentées dans ce rapport sont tirées de la phase 4 de l'enquête Track auprès des utilisateurs de drogues injectables au Canada. L'enquête Track auprès des utilisateurs de drogues injectables se base sur l'échantillonnage par lieu de rencontre : les participants sont recrutés dans les lieux où ils ont l'habitude de se réunir, le plus souvent, mais sans s'y limiter, où la prévention liée aux infections transmissibles sexuellement et par le sang, des services de dépistage et de traitement sont fournis, y compris des programmes de distribution d'aiguilles et de seringues. Les personnes qui avaient consommé des drogues injectables au cours des six mois précédant le recrutement et qui avaient l'âge minimal requis pour donner leur consentement, lequel a été déterminé à chaque site selon les exigences locales en matière d'éthique de la recherche, ont été autorisées à participer à l'enquête. Les participants admissibles et consentants ont rempli un questionnaire administré par un intervieweur et fourni un échantillon biologique de sang séché (ou un échantillon de salive dans le cas des sites du réseau de Surveillance des maladies infectieuses chez les utilisateurs de drogues par injection (SurvUDI).

Le protocole de surveillance et le questionnaire ont été approuvés par le Comité d'éthique de la recherche de Santé Canada et de l'Agence de la santé publique du Canada et, le cas échéant, par les comités d'éthique de la recherche de chaque site sentinelle. Les mêmes stratégies d'échantillonnage et de recrutement, ainsi que le même questionnaire de base (avec quelques révisions mineures) ont été employés lors des quatre phases pour permettre la comparabilité des résultats au fil du temps. Les méthodes d'enquête, la sélection des sites sentinelles, les détails du questionnaire et les algorithmes d'essai en laboratoire sont décrits de manière plus détaillée ailleurs (6).

\section{Questionnaire administré par un intervieweur et échantillon biologique}

Le questionnaire de l'enquête Track auprès des utilisateurs de drogues injectables vise à recueillir des renseignements sur les caractéristiques sociodémographiques, les déterminants sociaux de la santé, le recours aux services de santé et de prévention (incluant le dépistage), la consommation de drogues et les comportements d'injection, les pratiques sexuelles, ainsi que les soins et le traitement du VIH et de l'hépatite C. L'intervieweur est responsable de faire remplir le questionnaire et il faut environ 30 minutes pour le remplir. 
Les échantillons de sang séché ont été analysés pour y détecter la présence du VIH (anticorps et antigènes) et de l'hépatite $C$ (anticorps et l'acide ribonucléique [ARN]). Les participants n'ont pas été informés des résultats de leurs analyses en laboratoire, car aucun renseignement d'identification n'a été recueilli pour préserver l'anonymat des participants. II a été demandé aux sites sentinelles d'assurer un service de dépistage sur place, telles I'analyse de biologie délocalisée et la phlébotomie complète, pendant les périodes de recrutement pour que les participants n'ayant pas connaissance de leur statut infectieux puissent, s'ils le souhaitaient, se faire dépister. Lorsque les sites n'étaient pas en mesure d'assurer le dépistage sur place, les participants étaient dirigés vers des centres de dépistage ou des services de soins de santé de la région.

\section{Analyse}

Un partenariat a été établi entre le Réseau canadien autochtone du sida, I'Agence et un groupe consultatif composé d'un représentant de l'Association des femmes Inuites du Canada (Pauktuutit) et de personnes ayant une expérience vécue de la consommation de drogues injectables, du VIH ou de l'hépatite C. À l'aide de l'approche à double optique dans laquelle les visions du monde autochtone et occidental étaient respectées, le groupe consultatif s'est réuni régulièrement pendant six mois pour examiner et interpréter les résultats de l'enquête. En plus de rédiger cet article, le groupe consultatif a dégagé des constatations et des thèmes clés qui correspondent aux priorités d'action de la collectivité, en plus de préparer des infographies complémentaires pertinentes sur le plan culturel et destinées à la collectivité. Ces infographies étaient axées sur les indicateurs liés à l'accès aux services de réduction des risques et de soins de santé, y compris les soins et le traitement du VIH et de I'hépatite $C$ et la prophylaxie de préexposition et seront publiées par le Réseau canadien autochtone du sida à une date ultérieure.

Les statistiques descriptives ont été calculées avec SAS Enterprise Guide 7.1. Les faibles valeurs numériques ont été évaluées pour déterminer le risque d'identification des participants et incluses lorsque le risque de réidentification était jugé nul, conformément à la directive de l'Agence sur la collecte, I'utilisation et la diffusion de l'information sur la santé publique (Agence, 2013, document non publié). Les participants dont la réponse était «Non indiqué», «Je ne sais pas» ou "Refus» ont été exclus du dénominateur de chaque analyse d'indicateur.

\section{Résultats}

Au total, 2383 personnes étaient admissibles et ont accepté de participer à la phase 4 de l'enquête dans les 14 sites sentinelles suivants : Whitehorse (Yn), centre et nord de l'île de Vancouver (C.-B.), Prince Albert (Sask.), Regina (Sask.), Winnipeg (Man.),
Thunder Bay (Ont.), London (Ont.), Hamilton (Ont.), NouveauBrunswick, Terre-Neuve et quatre zones géographiques du réseau SurvUDI (Ottawa [Ont.] et région de l'Outaouais [Qc], Québec [Qc], Montréal [Qc] et autres sites urbains au Québec [Abitibi-Témiscamingue, Montérégie, Saguenay-Lac-Saint-Jean, Cantons de l'Est, Mauricie et Centre-du-Québec]).

Parmi les 2360 participants qui ont répondu à la question «Êtes-vous un autochtone, c'est-à-dire un membre des Premières Nations, Métis ou Inuit?», 997 (42,2\%) se sont identifiés comme autochtones. La proportion de participants autochtones dans chaque site sentinelle variait de moins de $10 \%$ dans trois sites SurvUDI (Montréal, Québec, Québec, Québec, autres sites urbains au Québec) à près de $80 \%$ à Whitehorse, à plus de $80 \%$ à Winnipeg et à Regina et à $95 \%$ à Prince Albert (tableau 1). Les 997 participants autochtones ont tous rempli un questionnaire et $884(88,7 \%)$ ont fourni un échantillon biologique.

\section{Tableau 1 : Proportion de participants autochtones et de participants d'autres ethnies par site sentinelle dans le cadre de l'enquête Track auprès des utilisateurs de drogues injectables au Canada, phase 4, 2017 à 2019 $(n=2383)$}

\begin{tabular}{|c|c|c|c|c|c|}
\hline \multirow[t]{2}{*}{ Site sentinelle } & \multicolumn{2}{|c|}{$\begin{array}{l}\text { Participants } \\
\text { autochtones }\end{array}$} & \multicolumn{2}{|c|}{$\begin{array}{l}\text { Participants } \\
\text { d'autres } \\
\text { ethnies } \\
\end{array}$} & \multirow[t]{2}{*}{ Total } \\
\hline & $\mathbf{n}$ & $\%$ & $\mathbf{n}$ & $\%$ & \\
\hline Whitehorse (Yn) & 39 & 79,6 & 10 & 20,4 & 49 \\
\hline $\begin{array}{l}\text { Centre et nord de l'île } \\
\text { de Vancouver (C.-B.) }\end{array}$ & 67 & 37,6 & 111 & 62,4 & 178 \\
\hline Prince Albert (Sask.) & 170 & 95,0 & 9 & 5,0 & 179 \\
\hline Regina (Sask.) & 174 & 84,9 & 31 & 15,1 & 205 \\
\hline Winnipeg (Man.) & 149 & 83,2 & 30 & 16,8 & 179 \\
\hline Thunder Bay (Ont.) & 137 & 68,8 & 62 & 31,2 & 199 \\
\hline London (Ont.) & 60 & 29,3 & 145 & 70,7 & 205 \\
\hline Hamilton (Ont.) & 38 & 25,2 & 113 & 74,8 & 151 \\
\hline $\begin{array}{l}\text { Ottawa (Ont.) et } \\
\text { région de l'Outaouais } \\
\text { (Qc) }\end{array}$ & 49 & 24,6 & 150 & 75,4 & 199 \\
\hline Montréal (Qc) & 16 & 8,0 & 184 & 92,0 & 200 \\
\hline Québec (Qc) & 11 & 8,9 & 113 & 91,1 & 124 \\
\hline $\begin{array}{l}\text { Autres sites urbains au } \\
\text { Québec }^{\mathrm{a}}\end{array}$ & 14 & 8,4 & 152 & 91,6 & 166 \\
\hline Nouveau-Brunswick & 29 & 14,6 & 170 & 85,4 & 199 \\
\hline Terre-Neuve & 44 & 34,6 & 83 & 65,4 & 127 \\
\hline Total & 997 & 42,2 & 1363 & 57,8 & 2360 \\
\hline
\end{tabular}




\section{Caractéristiques sociodémographiques}

Sur l'ensemble des participants de la phase $4,82,9 \%$ se sont identifiés comme membres des Premières Nations, 14,9\% comme membres des Métis et 2,2\% comme membres des Inuits. Parmi les participants autochtones, $13,8 \%$ ont déclaré vivre dans une collectivité des Premières Nations, des Métis ou des Inuits au moment de l'entrevue (tableau 2). Quatre sites sentinelles, trois dans les Prairies et un dans l'Ouest de l'Ontario, représentaient plus de $60 \%$ de tous les participants autochtones, tandis que la proportion de participants autochtones dans les autres sites sentinelles se situait entre $1 \%$ et $7 \%$.

Tableau 2 : Caractéristiques sociodémographiques des participants autochtones à l'enquête Track auprès des utilisateurs de drogues injectables au Canada, phase 4, 2017 à $2019(n=997)$

\section{Caractéristiques socio-démographiques ${ }^{a}$}

\begin{tabular}{|c|c|c|c|}
\hline \multirow{3}{*}{$\begin{array}{l}\text { Sous-groupe } \\
\text { autochtone }\end{array}$} & Premières Nations & 787 & 82,9 \\
\hline & Métis & 141 & 14,9 \\
\hline & Inuits & 21 & 2,2 \\
\hline \multicolumn{2}{|c|}{$\begin{array}{l}\text { Habitant d'une collectivité des Premières Nations, des Métis } \\
\text { ou des Inuits }\end{array}$} & 128 & 13,8 \\
\hline \multirow[t]{4}{*}{ Groupe d'âge } & Moins de 25 ans & 80 & 8,0 \\
\hline & $25-39$ ans & 463 & 46,5 \\
\hline & $40-54$ ans & 364 & 36,6 \\
\hline & 55 ans ou plus & 89 & 8,9 \\
\hline \multirow{4}{*}{$\begin{array}{l}\text { Identité de } \\
\text { genre }^{c}\end{array}$} & Homme cisgenre & 542 & 54,5 \\
\hline & Femme cisgenre & 426 & 42,9 \\
\hline & Personne transféminine ${ }^{d}$ & 14 & 1,4 \\
\hline & Personne transmasculine ${ }^{e}$ & 12 & 1,2 \\
\hline \multirow{5}{*}{$\begin{array}{l}\text { Orientation } \\
\text { sexuelle }\end{array}$} & Hétérosexuel & 850 & 85,7 \\
\hline & Bisexuel & 91 & 9,2 \\
\hline & Gai ou lesbienne & 26 & 2,6 \\
\hline & Bispirituel(le) & 17 & 1,7 \\
\hline & Autres $^{f}$ & 8 & 0,8 \\
\hline \multirow[t]{14}{*}{ Site sentinelle } & Regina (Sask.) & 174 & 17,5 \\
\hline & Prince Albert (Sask.) & 170 & 17,1 \\
\hline & Winnipeg (Man.) & 149 & 14,9 \\
\hline & Thunder Bay (Ont.) & 137 & 13,7 \\
\hline & $\begin{array}{l}\text { Centre et nord de l'île de Vancouver } \\
\text { (C.-B.) }\end{array}$ & 67 & 6,7 \\
\hline & London (Ont.) & 60 & 6,0 \\
\hline & $\begin{array}{l}\text { Ottawa (Ont.) et région de l'Outaouais } \\
\text { (Qc) }\end{array}$ & 49 & 4,9 \\
\hline & Terre-Neuve & 44 & 4,4 \\
\hline & Whitehorse (Yn) & 39 & 3,9 \\
\hline & Hamilton (Ont.) & 38 & 3,8 \\
\hline & Nouveau-Brunswick & 29 & 2,9 \\
\hline & Montréal (Qc) & 16 & 1,6 \\
\hline & $\begin{array}{l}\text { Autres sites urbains dans la province de } \\
\text { Québec }^{9}\end{array}$ & 14 & 1,4 \\
\hline & Québec (Qc) & 11 & 1,1 \\
\hline
\end{tabular}

Abréviations : C.-B., Colombie-Britannique; Man., Manitoba; Ont., Ontario; Qc, Québec Sask., Saskatchewan; Yn, Yukon

a Proportion variable de participants qui ont répondu à chacune des questions. Information manquante ou non recueillie pour $1 \%$ à $5 \%$ des caractéristiques sociodémographiques

${ }^{b}$ Cette question n'a pas été posée au site de London
Notes de bas de page du tableau suite

'La mesure multidimensionnelle du sexe et du genre (Multidimensional Sex/Gender Measure) a été utilisée pour mesurer l'identité de genre (7)

d Les personnes transféminines comprenaient les personnes à qui le sexe masculin à été assigné à la naissance et qui s'identifiaient au sexe féminin ou non binaire

Les personnes transmasculines comprenaient les personnes à qui le sexe féminin à été assigné à la naissance et qui s'identifiaient au sexe masculin ou non binaire

Autres, incluaient les personnes ayant répondu pansexuelles, exploratoires et autres réponses non classifiables

${ }^{9}$ Autres sites urbains dans la province de Québec, soit Abitibi-Témiscamingue, Montérégie Saguenay-Lac-Saint-Jean, Estrie et Mauricie-Centre du Québec

L'âge moyen des participants était de 38,9 ans. La majorité des participants étaient âgés de 25 à 39 ans (46,5\%), alors que les participants de 40 à 54 ans ne représentaient que $36,6 \%$ et que ceux de moins de 25 ans $(8,0 \%)$ ou de 55 ans ou plus $(8,9 \%)$ formaient les plus petites cohortes.

Dans cette phase, un peu plus de la moitié $(54,5 \%)$ des participants s'identifient comme hommes cisgenres, $42,9 \%$ comme femmes cisgenres et 1,4\% comme personnes transféminines (i.e. des personnes de sexe masculin à la naissance, mais qui s'identifient comme femmes ou non binaires) et 1,2 \% comme personnes transmasculines (i.e. des personnes de sexe féminin à la naissance, mais qui s'identifient comme hommes ou non binaires). Une grande proportion des participants ont déclaré avoir une orientation sexuelle hétérosexuelle $(85,7 \%)$ alors que des proportions plus faibles ont déclaré être bisexuels $(9,2 \%)$, gais ou lesbiennes $(2,6 \%)$, bispirituels $(1,7 \%)$ ou autres $(0,8 \%)$.

\section{Déterminants sociaux de la santé}

Parmi les participants de la phase 4, un peu plus de la moitié $(57,9 \%)$ avaient un niveau d'instruction égal ou inférieur au secondaire, $26,4 \%$ avaient terminé leurs études secondaires et $15,8 \%$ avaient réalisé des études supérieures après les études secondaires (tableau 3 ). Au cours des six mois précédant l'entrevue, un plus grand nombre de participants ont déclaré avoir été sans emploi (70,3\%) ou être bénéficiaires de l'aide sociale $(66,7 \%)$ ou recevoir une allocation d'invalidité $(33,6 \%)$. Une large proportion (83,7\%) de participants avaient rencontré des difficultés financières (du mal à «joindre les deux bouts») au cours des douze mois précédant l'entrevue.

Les deux tiers $(66,2 \%)$ des participants ont dit s'être trouvés dans une situation de logement précaire au cours des six mois précédant l'entrevue. Cela inclut avoir vécu dans une chambre d'hôtel ou de motel, une maison de chambre ou une pension de famille, un refuge ou une auberge de jeunesse, une maison ou un foyer de transition, un établissement psychiatrique ou un centre de désintoxication, un lieu public ou un établissement correctionnel. Dans l'ensemble, 75,2 \% des participants ont déclaré avoir déjà été incarcérés et 26,1 \% l'avaient été au cours des douze mois précédant l'entrevue.

Une large proportion de participants $(84,0 \%)$ ont déclaré que leur santé mentale était "passable à excellente» alors qu'une plus petite proportion, soit $16 \%$ des participants, a déclaré qu'elle était «mauvaise». Parmi les participants autochtones, $23,7 \%$ avaient fréquenté un pensionnat autochtone alors que 
Tableau 3 : Déterminants sociaux de la santé des participants à l'enquête Track auprès des utilisateurs de drogues injectables au Canada, phase 4, 2017 à 2019 $(\mathrm{n}=997)$

\begin{tabular}{|c|c|c|c|}
\hline \multicolumn{2}{|c|}{ Déterminants sociaux de la santéa } & $\mathbf{n}$ & $\%$ \\
\hline \multirow{3}{*}{$\begin{array}{l}\text { Niveau d'instruction } \\
\text { le plus élevé }\end{array}$} & $\begin{array}{l}\text { Niveau d'instruction égal ou inférieur au } \\
\text { secondaire }\end{array}$ & 575 & 57,9 \\
\hline & Diplôme d'études secondaires & 262 & 26,4 \\
\hline & $\begin{array}{l}\text { Études supérieures après les études } \\
\text { secondaires }\end{array}$ & 157 & 15,8 \\
\hline \multicolumn{2}{|c|}{ Difficultés financières ${ }^{b, c}$, au cours des douze derniers mois } & 707 & 83,7 \\
\hline \multirow{4}{*}{$\begin{array}{l}\text { Situation de } \\
\text { logement précaired }^{\text {, }} \\
\text { au cours des six } \\
\text { derniers mois }\end{array}$} & gement précaire & 659 & 66,2 \\
\hline & Situation de logement stable & 336 & 33,8 \\
\hline & Antécédents d'incarcératione, dans sa vie & 691 & 75,2 \\
\hline & $\begin{array}{l}\text { Incarcération, au cours des douze } \\
\text { derniers mois }{ }^{c}\end{array}$ & 224 & 26,1 \\
\hline \multirow{2}{*}{ Santé mentale $e^{f}$} & Passable à excellente & 756 & 84,0 \\
\hline & Mauvaise & 144 & 16,0 \\
\hline \multirow{6}{*}{$\begin{array}{l}\text { Autres déterminants } \\
\text { sociaux de la santé }\end{array}$} & $\begin{array}{l}\text { Antécédents de stigmatisation et de } \\
\text { discrimination }{ }^{c, g} \text {, dans sa vie }\end{array}$ & 753 & 90,2 \\
\hline & $\begin{array}{l}\text { Antécédents de stigmatisation et de } \\
\text { discrimination } n^{c, g} \text {, au cours des douze } \\
\text { derniers mois }\end{array}$ & 704 & 84,6 \\
\hline & $\begin{array}{l}\text { Antécédents de violences physiques, } \\
\text { sexuelles et/ou psychologiques durant }^{\text {l'enfance }}{ }^{c}\end{array}$ & 729 & 87,5 \\
\hline & $\begin{array}{l}\text { Antécédents de violences physiques, } \\
\text { sexuelles et/ou psychologiques de la } \\
\text { part d'un partenaire sexuel }{ }^{c}\end{array}$ & 654 & 78,6 \\
\hline & $\begin{array}{l}\text { Fréquentation d'un pensionnat } \\
\text { autochtone }^{c}\end{array}$ & 197 & 23,7 \\
\hline & $\begin{array}{l}\text { Membre de la famille ayant fréquenté un } \\
\text { pensionnat autochtone }{ }^{c}\end{array}$ & 687 & 89,8 \\
\hline \multicolumn{4}{|c|}{$\begin{array}{l}\text { a Proportion variable de participants qui ont répondu à chacune des questions. Information } \\
\text { manquante ou non recueillie pour } 1 \% \text { à } 10 \% \text { des déterminants sociaux de la santé } \\
\text { b Défini en tant qu'avoir eu du mal à «joindre les deux bouts » au cours de l'année précédant } \\
\text { l'entrevue } \\
\text { c Question non posée dans les sites du réseau SurvUDI et à London } \\
\text { ¿Défini en tant qu'avoir vécu dans une chambre d'hôtel ou de motel, une maison de chambre } \\
\text { ou une pension de famille, un refuge ou une auberge de jeunesse, une maison ou un foyer de } \\
\text { transition, un établissement psychiatrique ou un centre de désintoxication, un lieu public ou un } \\
\text { établissement correctionnel. Situation de logement stable comprend le fait de vivre dans un } \\
\text { appartement ou une maison ou dans l'appartement ou la maison d'un parent } \\
\text { e Seules des données partielles étaient disponibles dans les sites du réseau SurvUDI } \\
\text { ' Question non posée dans les sites du réseau SurvUDI } \\
\text { g Défini en tant qu'avoir déjà souffert de stigmatisation ou de discrimination (e.g. évitement, pitié, } \\
\text { blâme, honte, rejet, agression verbale ou intimidation) fondée sur l'origine ethnique ou culturelle, } \\
\text { le statut de séropositivité pour le VIH, le statut de séropositivité pour l'hépatite C, I'orientation } \\
\text { sexuelle, la consommation d'alcool ou de drogues ou le recours au travail du sexe }\end{array}$} \\
\hline
\end{tabular}

$89,8 \%$ d'entre eux comptaient dans leur famille une personne qui avait fréquenté un pensionnat autochtone.

La majorité des participants (90,2\%) ont déclaré avoir souffert de stigmatisation et de discrimination (liée à l'origine ethnique ou culturelle, au statut de séropositivité pour le $\mathrm{VIH}$, au statut de séropositivité pour I'hépatite $C$, à l'orientation sexuelle, à la consommation d'alcool ou de drogues ou au travail du sexe) dans leur vie alors que $84,6 \%$ d'entre eux ont dit en avoir vécu dans les douze mois précédant l'entrevue. Une large proportion de participants ont également souffert de violences physiques, sexuelles et/ou psychologiques durant l'enfance $(87,5 \%)$ ou de la part d'un partenaire sexuel $(78,6 \%)$.

\section{Accès aux soins de santé primaires et recours aux services de prévention et de dépistage}

Les participants ont été interrogés sur l'accès aux soins de santé primaires et sur le recours aux services de réduction des méfaits et de prévention des infections transmissibles sexuellement et par le sang, ainsi que sur les modes de dépistage du VIH et de I'hépatite $C$ (tableau 4). Dans l'ensemble, près des trois quarts $(72,2 \%)$ des participants avaient accès à des soins de santé primaires alors qu'une proportion légèrement plus faible $(63,9 \%)$ avait un fournisseur de soins de santé primaires régulier. Au cours des douze mois précédant l'entrevue, le quart des participants $(25,1 \%)$ ont utilisé des services de santé qui comprenaient des pratiques de santé ou de guérison autochtones comme un guérisseur traditionnel, un aîné de la collectivité, la Ligne d'écoute d'espoir pour le mieux-être (8) ou d'autres services de santé propres aux autochtones. Les services de conseil en santé mentale ont été utilisés par $28,5 \%$ des participants au cours des douze mois précédant l'entrevue.

\section{Tableau 4 : Accès et recours aux soins de santé, aux services de prévention et au dépistage du VIH et de l'hépatite $C$ chez les participants autochtones à l'enquête Track auprès des utilisateurs de drogues injectables au Canada, phase 4, 2017 à 2019 ( $n=997)$}

\begin{tabular}{|l|l|l|} 
Accès et recours aux services de soins de santéa & $\mathbf{n}$ & $\%$
\end{tabular}

\begin{tabular}{|c|c|c|}
\hline Accès aux soins de santé primaires ${ }^{b}$ & 594 & 72,2 \\
\hline Accès à un fournisseur de soins de santé primaires ${ }^{b}$ & 528 & 63,9 \\
\hline $\begin{array}{l}\text { Utilisation de services comprenant des pratiques de } \\
\text { santé ou de guérison autochtones, au cours des douze } \\
\text { derniers mois } \text { s }^{b, c}\end{array}$ & 206 & 25,1 \\
\hline $\begin{array}{l}\text { Recours aux services de conseil en santé mentale, au } \\
\text { cours des douze derniers mois }{ }^{d}\end{array}$ & 252 & 28,5 \\
\hline \multicolumn{3}{|l|}{ Recours aux services de prévention et de dépistage } \\
\hline $\begin{array}{l}\text { Utilisation d'un programme de distribution d'aiguilles et } \\
\text { de seringues, au cours des douze derniers mois }\end{array}$ & 800 & 90,5 \\
\hline Antécédents de dépistage du VIH, dans sa vie & 841 & 87,9 \\
\hline Antécédents de dépistage du VHC, dans sa vie & 833 & 87,8 \\
\hline $\begin{array}{l}\text { Conseils de prévention à propos des ITSS, au cours des } \\
\text { douze derniers mois }\end{array}$ & 429 & 54,2 \\
\hline $\begin{array}{l}\text { Recours à un programme de distribution de préservatifs, } \\
\text { au cours des douze derniers mois }{ }^{\mathrm{b}}\end{array}$ & 402 & 48,9 \\
\hline $\begin{array}{l}\text { Recours à la méthadone, au suboxone ou à tout autre } \\
\text { traitement de substitution aux opiacés, au cours des } \\
\text { douze derniers mois }{ }^{d}\end{array}$ & 385 & 43,6 \\
\hline $\begin{array}{l}\text { Recours aux services de traitement pour la } \\
\text { consommation de drogues ou d'alcool, au cours des } \\
\text { douze derniers mois } \text { se }^{\text {,e }}\end{array}$ & 224 & 27,2 \\
\hline $\begin{array}{l}\text { Fréquentation d'un site d'injection ou de consommation } \\
\text { supervisée, au cours des douze derniers mois }{ }^{d}\end{array}$ & 88 & 9,9 \\
\hline \multicolumn{3}{|c|}{$\begin{array}{l}\text { Sensibilisation aux prophylaxies préexposition et post-exposition } \\
\text { non professionnelle }\end{array}$} \\
\hline $\begin{array}{l}\text { Sensibilisation à la prophylaxie préexposition au VIH par } \\
\text { voie orale }^{d}\end{array}$ & 98 & 11,5 \\
\hline $\begin{array}{l}\text { Sensibilisation à la prophylaxie post-exposition non } \\
\text { professionnelle au } \mathrm{VIH}^{\mathrm{d}}\end{array}$ & 88 & 1 \\
\hline
\end{tabular}


Notes de bas de page du tableau suite

Abréviations: ITTS, infections transmissibles sexuellement et par le sang; VHC, virus de l'hépatite $\mathrm{C} ; \mathrm{VIH}$, virus de l'immunodéficience humaine

Proportion variable de participants qui ont répondu à chacune des questions. Information

manquante ou non recueillie pour $2 \%$ à $10 \%$ de ces indicateurs

b Question non posée dans les sites du réseau SurvUDI et à London

Les pratiques autochtones de santé ou de guérison comprennent un guérisseur traditionnel, un aîné de la collectivité, la Ligne d'écoute d'espoir pour le mieux-être ou d'autres services de santé propres aux autochtones

d Question non posée dans les sites du réseau SurvUD

e Comprend des services comme les traitements dans des centres, le counselling de groupe ou un guérisseur traditionnel

Le recours aux services de réduction des méfaits et de prévention des infections transmissibles sexuellement et par le sang au cours des douze mois précédant l'entrevue varie selon le service (tableau 4). La majorité des participants $(90,5 \%$ ) a déclaré utiliser un programme de distribution d'aiguilles et de seringues alors qu'une faible proportion (9,9\%) a utilisé un site d'injection ou de consommation supervisé. Moins de la moitié des participants $(43,6 \%)$ ont utilisé de la méthadone, du suboxone ou un autre traitement de substitution aux opioïdes et un peu plus du quart des participants $(27,2 \%)$ ont utilisé des services de traitement pour la consommation de drogues ou d'alcool au cours des douze mois précédant l'entrevue. Au cours de la même période, $48,9 \%$ ont déclaré utiliser un programme de distribution de préservatifs et $54,2 \%$ ont reçu des conseils en prévention des infections transmissibles sexuellement et par le sang. Une grande proportion des participants ont déclaré avoir déjà subi un test de dépistage du VIH $(87,9 \%)$ et de l'hépatite $\mathrm{C}$ $(87,8$ \%) (tableau 4).

Seule une faible proportion des participants étaient au courant de la prophylaxie post-exposition au VIH par voie orale (11,5\%) et de la prophylaxie post-exposition non professionnelle contre le $\mathrm{VIH}(10,8 \%)$ (tableau 4). Au cours des douze mois précédant l'entrevue, 45,7\% des participants ont évité les services de santé et parmi ceux qui n'avaient jamais été testés pour le VIH et ceux qui ont déclaré être séronégatifs, $23,1 \%$ ont évité de se faire dépister pour le VIH en raison de la stigmatisation et de la discrimination (défini comme la peur ou l'inquiétude d'être victime de stigmatisation ou de discrimination de la part du personnel ou de voisins, la peur ou l'inquiétude de quelqu'un qui pourrait apprendre qu'il s'injecte des drogues, la peur ou l'inquiétude d'être victime de violence ou l'expérience de celui-ci, la peur ou l'inquiétude d'être victime de harcèlement ou d'arrestation de la part de la police ou l'expérience de celui-ci).

\section{Comportements d'injection}

L'âge moyen auquel les participants ont déclaré avoir consommé des drogues injectables pour la première fois était de 24,5 ans. Moins de la moitié de tous les participants (40,5\%) ont déclaré s'être injecté quotidiennement au cours du mois précédant l'entrevue et un peu plus de la moitié $(53,5 \%)$ ont déclaré s'être injectés dans un lieu public au cours des six mois précédant l'entrevue. Dans l'ensemble, 93,1\% des participants ont utilisé une aiguille et une seringue stériles lors de la dernière injection. Au cours des six mois précédant l'entrevue, 10,0\% des participants se sont injectés avec des aiguilles ou des seringues usagées et parmi eux, une majorité de participants $(85,0 \%)$ empruntaient des aiguilles ou des seringues à des personnes qu'ils connaissaient bien (e.g. membres de la famille, amis ou partenaires sexuels). Un peu moins de la moitié $(45,7 \%)$ se sont injectés avec du matériel d'injection usagé autre que des aiguilles ou des seringues, par exemple, de l'eau, des filtres, des réchauds, des garrots, de la ouate ou des acidifiants au cours des six mois précédant l'entrevue. Parmi ceux qui empruntaient du matériel d'injection usagé (autre que des aiguilles ou des seringues), la majorité $(85,9 \%)$ a déclaré l'avoir emprunté auprès de personnes qu'ils connaissaient bien (membres de la famille, amis ou partenaires sexuels). Plus de la moitié des participants (58,3 \%) avaient emprunté du matériel de consommation usagé autre que du matériel d'injection tel que des pailles, des billets de banque ou des pipes au cours des six mois précédant l'entrevue (tableau 5).

Tableau 5 : Comportements d'injection des participants autochtones à l'enquête Track auprès des utilisateurs de drogues injectables au Canada, phase 4, 2017 à 2019 $(n=997)$

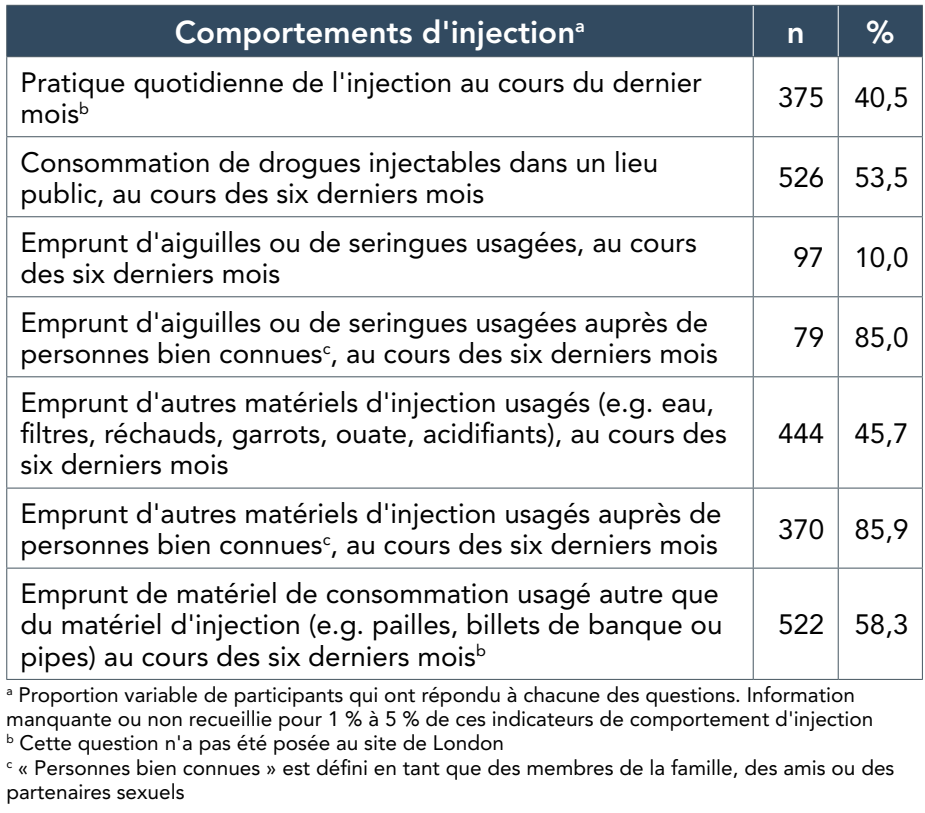

\section{Consommation de drogues et antécédents de surdose}

Parmi les participants autochtones, la cocaïne était la drogue la plus fréquemment injectée au cours des six mois précédant l'entrevue (58,2\%), suivie de la méthamphétamine $(55,5 \%)$, de la morphine $(49,7 \%)$ de l'hydromorphone $(43,8 \%)$ et de l'héroïne $(30,4 \%)$ (tableau 6). Environ 20 à $30 \%$ des participants se sont injectés du Ritalin seul (29,3\%), du fentanyl (23,4\%), du crack $(22,9 \%)$, des amphétamines $(20,7 \%)$ ou de l'oxycodone $(18,7 \%)$.

Un large éventail de drogues non injectables ont été utilisées au cours des six mois précédant l'entrevue, le cannabis (71,9\%), l'alcool (66,2 \%), la méthamphétamine (51,3\%), la cocaïne 
Tableau 6 : Consommation de drogues et antécédents de surdose des participants à l'enquête Track auprès des utilisateurs de drogues injectables au Canada, phase 4, 2017 à $2019(n=997)$

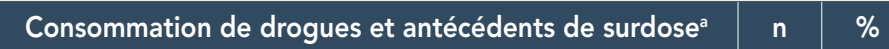
Cinq drogues par injection les plus utilisées, au cours des six derniers mois ${ }^{b}$

\begin{tabular}{|l|r|r|}
\hline Cocaïne & 576 & 58,2 \\
\hline Méthamphétamine & 548 & 55,5 \\
\hline Morphine & 491 & 49,7 \\
\hline Hydromorphone & 433 & 43,8 \\
\hline Héroïne & 299 & 30,4 \\
\hline Cinq drogues sans injection les plus utilisées, au cours des six derniers mois \\
\hline Cannabis & 708 & 71,9 \\
\hline Alcool & 652 & 66,2 \\
\hline Méthamphétamine & 503 & 51,3 \\
\hline Cocaïne & 490 & 49,9 \\
\hline Crack & 479 & 48,8 \\
\hline
\end{tabular}

Connaissance, accessibilité et utilisation d'une trousse contre les surdoses

Connaissance de l'existence des trousses contre les surdoses ${ }^{c}$

$693 \quad 83,3$

Antécédents d'utilisation d'une trousse contre les surdoses ${ }^{c, d}$

$178 \quad 25,7$

Accessibilité des trousses contre les surdoses dans la collectivité des participants ${ }^{d, e}$

\begin{tabular}{|l|r|r|}
\hline Non & 28 & 4,0 \\
\hline Oui & 631 & 91,1 \\
\hline Ne sait pas & 34 & 4,9 \\
\hline
\end{tabular}

\section{Expériences de surdose}

Antécédents de surdose au cours des six derniers mois ${ }^{\mathrm{d}, \mathrm{f}}$

$185 \quad 20,9$

Cinq drogues ou substances les plus couramment utilisées lors de la dernière surdose $e^{b, c}$

\begin{tabular}{|l|r|r|}
\hline Fentanyl & 67 & 42,7 \\
\hline Héroïne & 67 & 41,6 \\
\hline Méthamphétamine & 50 & 30,7 \\
\hline Alcool & 33 & 21,5 \\
\hline Cocaïne & 33 & 20,3 \\
\hline
\end{tabular}

a Proportion variable de participants qui ont répondu à chacune des questions. Information manquante ou non recueillie pour $1 \%$ à $2 \%$ de ces indicateurs, sauf pour les drogues utilisées lors de la dernière surdose pour lesquelles des informations étaient manquantes ou non recueillies pour $10 \%$ à $15 \%$ de ces indicateurs

b Les participants ont indiqué toutes les drogues (qu'ils avaient injectées, consommées ou utilisées lors de la dernière surdose) à des fins non médicales au cours des six mois précédant l'entrevue. Les drogues les plus fréquemment citées par les participants sont présentées. Les réponses ne s'excluent pas mutuellement

' Question non posée dans les sites du réseau SurvUDI et à London

d Parmi les participants qui avaient entendu parler des trousses contre les surdoses

- Question non posée dans les sites du réseau SurvUDI

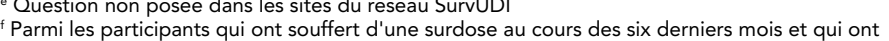
fourni une réponse

$(49,9 \%)$ et le crack $(48,8 \%)$ ayant été les drogues les plus souvent utilisées. La consommation d'analgésiques opioïdes (non injectables) a également été déclarée spécifiquement pour la codéine $(34,7 \%)$, la méthadone $(31,0 \%)$, la morphine $(30,9 \%)$ et I'hydromorphone (27,3\%) (tableau 6).
La plupart des participants avaient entendu parler des trousses contre les surdoses $(83,3 \%)$ et une majorité d'entre eux $(91,1 \%)$ ont déclaré y avoir accès dans leur collectivité. Parmi les participants qui avaient entendu parler des trousses contre les surdoses, le tiers (33,8 \%) des participants autochtones en avaient une avec eux et le quart (25,7\%) en avaient déjà utilisé une sur quelqu'un d'autre. Au cours des six mois précédant l'entrevue, 20,9\% des participants ont souffert d'une surdose et les drogues les plus souvent utilisées lors de la dernière surdose étaient le fentanyl $(42,7 \%)$, I'héroïne $(41,6 \%)$ et la méthamphétamine $(30,7 \%)$ (tableau 6$)$.

\section{Comportements sexuels à risque}

Parmi les participants ayant des antécédents de relations sexuelles, 35,4 \% avaient eu deux partenaires sexuels ou plus au cours des six mois précédant l'entrevue (tableau 7). Parmi les participants qui avaient un partenaire sexuel régulier, 85,6\% ont déclaré une utilisation irrégulière du préservatif pendant les relations sexuelles vaginales et/ou anales. Parmi les participants qui avaient un partenaire sexuel occasionnel, 57,6\% ont déclaré une utilisation incohérente du préservatif pendant les relations sexuelles vaginales et/ou anales. Une faible proportion (16,0\%) avait eu des relations sexuelles rémunérées au moins une fois, parmi lesquelles $26,3 \%$ avaient eu des relations sexuelles sans condom lors de leur dernière relation sexuelle rémunérée (tableau 7). La plupart des participants (81,6\%) ont déclaré consommer des substances avant ou pendant les relations sexuelles (tableau 7).

\section{Tableau 7 : Comportements sexuels des participants autochtones à l'enquête Track auprès des utilisateurs de drogues injectables au Canada, phase 4, 2017 à 2019} $(n=997)$

\begin{tabular}{|c|c|c|}
\hline Comportements sexuels à risque ${ }^{a}$ & n & $\%$ \\
\hline $\begin{array}{l}\text { Deux partenaires sexuels ou plus, au cours des six } \\
\text { derniers mois }^{b}\end{array}$ & 330 & 35,4 \\
\hline $\begin{array}{l}\text { Utilisation irrégulière du préservatif lors de relations } \\
\text { vaginales ou anales avec un partenaire sexuel } \\
\text { habituel, au cours des six derniers mois }{ }^{c}\end{array}$ & 540 & 85,6 \\
\hline $\begin{array}{l}\text { Utilisation irrégulière du préservatif lors de relations } \\
\text { vaginales ou anales avec un partenaire sexuel } \\
\text { occasionnel, au cours des six derniers mois }{ }^{c}\end{array}$ & 167 & 57,6 \\
\hline $\begin{array}{l}\text { Relations sexuelles rémunérées, au cours des six } \\
\text { derniers mois }\end{array}$ & 127 & 16,0 \\
\hline $\begin{array}{l}\text { Absence de préservatif lors de la dernière relation } \\
\text { sexuelle rémunérée }^{d}\end{array}$ & 31 & 26,3 \\
\hline $\begin{array}{l}\text { Consommation de substances avant ou pendant les } \\
\text { relations sexuelles au cours des six derniers mois }{ }^{d}\end{array}$ & 586 & 81,6 \\
\hline \multicolumn{3}{|c|}{$\begin{array}{l}\text { a Proportion variable de participants qui ont répondu à chacune des questions. Information } \\
\text { manquante ou non recueillie pour } 2 \% \text { à } 14 \% \text { de ces indicateurs de comportements sexuels à } \\
\text { risque } \\
\text { b Parmi les participants ayant des antécédents de relations sexuelles } \\
\text { c Utilisation irrégulière du préservatif est défini comme ne pas systématiquement utiliser un } \\
\text { préservatif (i.e. jamais, parfois ou souvent) lors des relations sexuelles. Cette question n'a pas été } \\
\text { posée au site de London } \\
{ }^{d} \text { Question non posée dans les sites du réseau SurvUDI }\end{array}$} \\
\hline
\end{tabular}




\section{Prévalence du VIH et de l'hépatite $\mathrm{C}$ et connaissance du statut d'infection à ces maladies}

Parmi les participants ayant fourni un échantillon biologique en quantité suffisante pour effectuer des dépistages $(n=879)$, des $15,4 \%$ qui étaient séropositifs pour le $\mathrm{VIH}, 78,2 \%$ étaient au courant de leur séropositivité (tableau 8). L'exposition à vie à l'infection par l'hépatite $C$ (i.e. la proportion de répondants séropositifs à l'hépatite $C$ ) était de $65,8 \%$ (parmi 863 échantillons en quantité suffisante pour le dépistage). Plus du tiers $(36,4 \%)$ étaient séropositifs pour l'ARN du virus de I'hépatite C (parmi 696 échantillons en quantité suffisante pour le dépistage), ce qui est un indicateur de l'infection actuelle à I'hépatite $C$, et $49,4 \%$ d'entre eux étaient au courant de leur séropositivité à l'ARN de l'hépatite $C$. Parmi les participants qui ont fourni un échantillon biologique en quantité suffisante pour le dépistage des anticorps du VIH et de l'ARN du virus de I'hépatite $\mathrm{C}, 6,0 \%$ étaient séropositifs pour le $\mathrm{VIH}$ et séropositifs pour l'ARN du virus de l'hépatite $C$.

Tableau 8 : Prévalence et cascade de soins du VIH et de l'hépatite $C$ et connaissance du statut d'infection à ces maladies chez les participants à l'enquête Track auprès des utilisateurs de drogues injectables au Canada, phase 4, 2017 à $2019(n=997)$

Prévalence du VIH et de l'hépatite $\mathrm{C}^{\mathrm{a}}$

\begin{tabular}{l|l} 
n & $\%$
\end{tabular}

Prévalence du VIH et connaissance du statut d'infection à la maladie

\begin{tabular}{|l|l|l|}
\hline Prévalence du $\mathrm{VIH}^{\mathrm{b}, \mathrm{c}}$ & 135 & 15,4 \\
\hline Connaissance du statut de séropositivité pour le $\mathrm{VIH}^{\mathrm{d}}$ & 104 & 78,2 \\
\hline
\end{tabular}

Cascade de soins du VIH (parmi les participants ayant connaissance de leur statut de séropositivité pour le VIH, $n=104$ )

Orientation vers les soins et les services liés au VIHe $100 \quad 96,2$

Suivi actuel d'un TA 8783,7

Observance du TA, sans aucune dose omise au cours du dernier mois ${ }^{\dagger}$

$30 \quad 43,5$

Autodéclaration d'une charge virale indétectable pour le $\mathrm{VIH}^{\mathrm{g}}$

Évitement des services liés au VIH en raison

d'antécédents de stigmatisation et de discrimination, au

cours des douze derniers mois

Évitement du traitement lié au VIH en raison

d'antécédents de stigmatisation et de discrimination, au

cours des douze derniers mois ${ }^{\uparrow}$

$47 \quad 64,4$

Prévalence de l'hépatite $C$ et connaissance du statut d'infection à la maladie

Prévalence des anticorps anti-VHC, ${ }^{c h}$

$568 \quad 65,8$

Prévalence de I'ARN du VHCc,i

$253 \quad 36,4$

Connaissance du statut de séropositivité pour l'ARN de I'hépatite $\mathrm{C}^{\mathrm{i}}$

12249,4

Cascade de soins de l'hépatite $C$ (parmi les participants ayant connaissance de leur statut de séropositivité pour l'ARN de I'hépatite $\mathrm{C}, \mathrm{n}=122$ )

Orientation vers les soins pour l'hépatite $C^{k}$

$66 \quad 54,1$

Antécédents de traitement contre l'hépatite $C$

1714,1

Tableau 8 : Prévalence et cascade de soins du VIH et de I'hépatite $C$ et connaissance du statut d'infection à ces maladies chez les participants à l'enquête Track auprès des utilisateurs de drogues injectables au Canada, phase 4, 2017 à $2019(\mathrm{n}=997)$ (suite)

\begin{tabular}{|c|c|c|}
\hline Prévalence du VIH et de l'hépatite Ca & $\mathbf{n}$ & $\%$ \\
\hline \multicolumn{3}{|l|}{$\begin{array}{l}\text { Cascade de soins de l'hépatite } C \text { (parmi les participants ayant } \\
\text { connaissance de leur statut de séropositivité pour l'ARN de } \\
\text { l'hépatite } C, n=122 \text { ) (suite) }\end{array}$} \\
\hline Suivi actuel d'un traitement contre l'hépatite $C^{\prime}$ & 7 & 5,8 \\
\hline \multicolumn{3}{|l|}{ Co-infection au VIH et à l'hépatite $\mathrm{C}^{\mathrm{m}}$} \\
\hline $\begin{array}{l}\text { Séropositivité pour le VIH et séropositivité pour l'ARN de } \\
\text { l'hépatite C }\end{array}$ & 42 & 6,0 \\
\hline
\end{tabular}

Abréviations : ARN, acide ribonucléique; TA, traitement antirétroviral; $\mathrm{VHC}$, virus de l'hépatite $\mathrm{C}$; $\mathrm{VIH}$, virus de l'immunodéficience humaine

a Proportion variable de participants qui ont répondu à chacune des questions. Information manquante ou non recueillie pour moins de $1 \%$ de ces indicateurs, sauf pour l'observance du TA (20\%) et l'autodéclaration d'une charge virale indétectable pour le VIH (26\%)

Parmi les participants ayant fourni un échantillon biologique en quantité suffisante pour détecter la présence du VIH $(n=879)$

c Les algorithmes de dépistage du VIH et de l'hépatite $\mathrm{C}$ sont présentés à l'annexe 1

d Parmi les participants chez qui la présence des anticorps anti-VIH a été détectée et qui ont déclaré leur diagnostic d'infection au VIH. Les participants ayant déclaré que les résultats de leur dernier test de dépistage du VIH étaient positifs et dont la séropositivité pour le VIH a été confirmée à la suite de l'analyse de l'échantillon biologique fourni au moment de l'entrevue ont été classés comme ayant connaissance de leur statut de séropositivité pour le $\mathrm{VIH}$

e Défini comme étant suivi par un médecin ou un fournisseur de soins de santé pour des services liés au $\mathrm{VIH}$ au moment de l'entrevue (au cours des six mois précédant l'entrevue dans le cas des sites du réseau SurvUDI et du site de London)

† Question non posée dans les sites du réseau SurvUDI et à London. Le dénominateur exclut les participants pour lesquels il manque des données

g Parmi les participants suivant un TA au moment de l'entrevue. Cette question n'a pas été posée dans les sites du réseau SurvUDI. Le dénominateur exclut les participants pour lesquels il manque des données

$\mathrm{h}$ Parmi les participants ayant fourni un échantillon biologique en quantité suffisante pour détecter la présence des anticorps anti-VHC $(n=863)$

i Parmi les participants ayant fourni un échantillon biologique en quantité suffisante pour détecter la présence des anticorps anti-VHC et de l'ARN du VHC. Les analyses visant à détecter la

présence de l'ARN du VHC n'ont pas été réalisées dans les sites du réseau SurvUDI $(n=696)$ présence de l'ARN du VHC n'ont pas été réalisées dans les sites du réseau SurvUDI $(n=696)$ j Parmi les participants chez qui la présence de l'ARN du VHC a été détectée et qui ont dé
leur statut actuel de séropositivité pour l'hépatite C. Les participants ayant déclaré être leur statut actuel de séropositivité pour l'hépatite $C$. Les participants ayant déclaré être confirmée à la suite de l'analyse de l'échantillon biologique fourni au moment de l'entrevue ont été classés comme ayant connaissance de leur statut de séropositivité pour l'ARN de l'hépatite $C$ Défini comme étant suivi par un médecin ou un fournisseur de soins de santé pour des services liés à l'hépatite $C$ au moment de l'entrevue. Le dénominateur exclut les participants pour lesquels lies à l'hepatite $\mathrm{C}$ au $\mathrm{m}$

' Le dénominateur exclut les participants pour lesquels il manque des données

$m$ Parmi les participants ayant fourni un échantillon biologique en quantité suffisante pour détecter la présence des anticorps anti-VIH et de l'ARN du VHC. Les analyses visant à détecter la présence de l'ARN du VHC n'ont pas été réalisées dans les sites du réseau SurvUDI

\section{Cascade de soins du VIH et de l'hépatite C}

Les indicateurs de la cascade de soins du VIH ont été mesurés parmi les participants autochtones ayant connaissance de leur statut de séropositivité pour le VIH (tableau 8). La majorité d'entre eux $(96,2 \%)$ étaient suivis par un médecin ou un fournisseur de soins de santé pour des services liés au VIH au moment de l'entrevue. Une grande partie $(83,7 \%)$ d'entre eux suivait un traitement antirétroviral au moment de l'entrevue. L'observance du traitement antirétroviral, mesurée par la prise systématique des doses prescrites au cours du mois précédant l'entrevue, était de 43,5\%. Parmi les participants qui suivaient un traitement antirétroviral au moment de l'entrevue, 64,4\% ont déclaré une charge virale indétectable pour le VIH. Environ le quart des participants qui avaient connaissance de leur statut de séropositivité pour le VIH ont déclaré éviter les services liés au VIH (25,3\%) ou le traitement du VIH $(21,7 \%)$ en raison d'antécédents de stigmatisation et de discrimination au cours des douze derniers mois précédant l'entrevue. 
Les indicateurs de la cascade de soins du VIH ont été mesurés parmi les participants autochtones ayant connaissance de leur séropositivité pour l'hépatite $C$ (tableau 8 ). Plus de la moitié $(54,1 \%)$ des participants ont déclaré avoir été orientés vers des soins pour l'hépatite $C_{\text {; }}$ une proportion plus faible $(10,6 \%)$ avait des antécédents de traitement contre l'hépatite $C$; une proportion encore plus faible $(5,8 \%)$ suivait actuellement un traitement contre I'hépatite $C$. Parmi les obstacles fréquents au fait de ne pas suivre de traitement pour l'hépatite $C$, mentionnons le fait que les participants consommaient de l'alcool ou des drogues (29,7\%), qu'ils n'ont commencé à recevoir des soins médicaux contre l'hépatite $C$ que récemment $(23,0 \%)$ ou que leur médecin leur a conseillé de retarder le traitement $(19,0 \%)$.

\section{Discussion}

La mise sur pied d'un groupe consultatif dirigé par des autochtones était fondamentalement importante et nécessaire pour l'analyse et l'interprétation des résultats de la surveillance axée sur les participants autochtones. La composition de l'équipe et l'utilisation de l'approche de vision à double optique respectaient les points de vue autochtones et occidentaux tout en favorisant la participation significative de différents intervenants, y compris des autochtones ayant vécu ou vivant une expérience de consommation de drogues injectables. La nature collaborative du groupe consultatif est une étape vers la réconciliation.

Une grande proportion des participants autochtones (84,0\%) ont déclaré que leur état de santé mentale allait de passable à excellent, une constatation qui ressort comparativement aux proportions déclarées pour d'autres indicateurs associés à une mauvaise santé mentale, soit la violence faite aux enfants et aux partenaires sexuels ( $87,5 \%$ et $78,6 \%$, respectivement); un membre de la famille ayant fréquenté un pensionnat autochtone (89,8 \%); incarcération (75,2\%); une situation de logement précaire $(66,2 \%)$ et le fait d'avoir déjà été victime de stigmatisation et de discrimination (90,2\%). Les niveaux élevés de santé et de bien-être mental déclarés peuvent refléter la résilience des peuples autochtones dans l'expérience individuelle et collective de traumatisme.

En ce qui concerne les indicateurs de prévention, des taux élevés de tests de dépistage du VIH et de l'hépatite $\mathrm{C}$ au cours de la vie ont été remarqués $(87,9 \%$ et $87,8 \%$ respectivement) alors que la majorité des participants $(90,5 \%)$ avaient utilisé les services d'un programme de distribution d'aiguilles et de seringues et déclaré avoir des pratiques d'injection sécuritaires $(93,1 \%$ ont déclaré avoir utilisé une aiguille et une seringue propres lors de la dernière injection). Le recours à d'autres services de réduction des méfaits était particulièrement plus faible, notamment le traitement de substitution aux opioïdes (43,6\%), les services de désintoxication $(27,2 \%)$, le programme de distribution de préservatifs $(48,9 \%)$ et les conseils sur les infections transmissibles sexuellement et par le sang (54,2\%). Bien que le recours à un site d'injection ou de consommation supervisé dans les douze mois précédant l'entrevue ait été faible $(9,9 \%)$, il faut noter que ce service n'est pas offert uniformément partout au Canada. La sensibilisation aux prophylaxies préexposition et post-exposition non professionnelle était faible $(11,5 \%$ et $10,8 \%$ respectivement). La plupart des participants avaient entendu parler des trousses de naloxone (83,3\%). Les proportions moins élevées de personnes ayant déclaré avoir une trousse pour surdose $(33,8 \%)$ témoignent du besoin continu d'accroître la distribution de la trousse de naloxone.

Parmi les participants autochtones à l'enquête Track auprès des utilisateurs de drogues injectables, la séroprévalence du VIH $(15,4 \%)$, I'exposition à vie à l'hépatite $C(65,8 \%)$ et l'infection actuelle à l'hépatite $C(36,4 \%)$ étaient élevées. Ces résultats corroborent ceux provenant d'autres études régionales qui soulignent l'incidence disproportionnée de la consommation de drogues injectables et du VIH et de l'hépatite $\mathrm{C}$ sur les peuples et les collectivités autochtones du Canada (9-12). Les indicateurs cibles du VIH 90-90-90 mesurés chez les autochtones qui consomment des drogues injectables dans cette enquête $(78,2 \%$ savent qu'ils sont séropositifs, $83,7 \%$ suivent actuellement un traitement antirétroviral et $64,4 \%$ déclarent une charge virale indétectable) sont encourageants, mais ces résultats indiquent qu'il faut améliorer l'accès aux soins et au traitement du VIH. De plus, les indicateurs de soins et de traitement de l'hépatite $C$ (i.e. $54,1 \%$ liés aux soins, 5,8 \% actuellement sous traitement) étaient considérablement inférieurs à ceux du $\mathrm{VIH}$, ce qui indique des lacunes importantes dans le dépistage, les soins et le traitement de l'hépatite $\mathrm{C}$ dans cette population clé.

\section{Prochaines étapes}

Les peuples et les collectivités autochtones sont débrouillards et résilients. Le lien avec la culture, la terre et les cérémonies les a aidés à comprendre la santé et à réagir individuellement et collectivement aux traumatismes historiques et continus comme le colonialisme et l'expérience des pensionnats. Alors que les peuples et les collectivités autochtones font face à des problèmes de santé continus comme les infections par le $\mathrm{VIH}$ et l'hépatite $\mathrm{C}$, le fardeau de la crise des opioïdes et les décès liés à des surdoses d'autres drogues mettent en évidence le besoin continu d'avoir accès à des services de prévention et de traitement culturellement pertinents, y compris la distribution accrue de trousses de naloxone contre les surdoses. Des interventions de prévention sont justifiées, comme une éducation complète sur la santé sexuelle et les infections transmissibles sexuellement et par le sang, ce qui inclut une plus grande sensibilisation et l'accès à la prophylaxie préexposition et post-exposition non professionnelle chez les personnes séronégatives au $\mathrm{VIH}$ à risque élevé d'infection afin de réduire d'être infectées (13). Un engagement continu dans l'interprétation des résultats de la surveillance chez les participants autochtones dans le cadre de réseaux propres 
aux autochtones, de guérisseurs traditionnels et d'approches communautaires peut également contribuer à la résilience des peuples et des collectivités autochtones.

\section{Forces et faiblesses}

Ce système national intégré de biosurveillance fournit de I'information sur le VIH et l'hépatite $\mathrm{C}$ chez les utilisateurs de drogues injectables afin que cette information puisse être utilisée aux niveaux local, provincial et fédéral pour éclairer et orienter les interventions en santé publique dans cette population. L'enquête Track auprès des utilisateurs de drogues injectables repose sur l'échantillonnage non probabiliste; il se peut donc que les résultats ne soient pas représentatifs de tous les utilisateurs de drogues injectables d'un site donné ou de l'ensemble du Canada. Un petit nombre de participants qui se sont identifiés comme Métis et Inuits, ainsi que ceux dont l'identité de genre était transmasculine ou transfémineuse, ont empêché les analyses effectuées pour les sous-groupes particuliers de voir les liens avec d'autres caractéristiques et indicateurs sociodémographiques. À l'exception des résultats de laboratoire, ces résultats sont fondés sur des questionnaires administrés par un intervieweur et sur des données autodéclarées; il est donc possible que certains comportements à risque aient été surreprésentés ou sous-représentés.

\section{Conclusion}

Les efforts communs du groupe consultatif dirigé par des autochtones ont facilité le leadership communautaire et l'analyse collaborative de l'enquête Track auprès des utilisateurs de drogues injectables. Cette collaboration a permis d'élaborer des produits du savoir qui diffuseront les résultats propres aux autochtones et les contextualiseront afin qu'ils soient les plus pertinents pour les intervenants dans divers contextes. Ces résultats de surveillance mettent en évidence les défis liés à l'accès et au maintien de soins et de traitements efficaces contre le $\mathrm{VIH}$ et l'hépatite $\mathrm{C}$ chez les autochtones qui utilisent des drogues injectables au Canada. Ces renseignements sont particulièrement importants pour éclairer les stratégies de réduction des méfaits et les services de prévention et de traitement des infections transmissibles sexuellement et par le sang propres aux autochtones au Canada. Un examen plus approfondi des obstacles et des facteurs qui facilitent l'accès et l'utilisation des services de prévention et de traitement des infections transmissibles sexuellement et par le sang et de réduction des méfaits est justifié.

\section{Déclaration des auteurs}

J. T. - Conceptualisation, analyse formelle, méthodologie, administration de projet, rédaction (version initiale, révision et édition)

M. S. - Conceptualisation, rédaction (version initiale, révision et édition)

D. B. - Conceptualisation, rédaction (version initiale, révision et édition)
C. H. - Conceptualisation, rédaction (version initiale, révision et édition)

M. M. - Conceptualisation, rédaction (version initiale, révision et édition)

T. S. - Conceptualisation, rédaction (version initiale, révision et édition)

F. C. - Conceptualisation, méthodologie, rédaction (révision et édition)

L. J. - Conceptualisation, conservation des données, analyse formelle, rédaction (version initiale, révision et édition)

M. B. - Conceptualisation, méthodologie, administration de projet, rédaction (révision et édition)

D. P. - Conceptualisation, acquisition du financement, méthodologie, rédaction (version initiale, révision et édition) R. M. - Conceptualisation, rédaction (version initiale, révision et édition)

\section{Intérêts concurrents}

Aucun.

\section{Remerciements}

La mise en place du système de surveillance Track a été rendue possible grâce à une collaboration fructueuse entre l'Agence de la santé publique du Canada (y compris le Laboratoire national de microbiologie) et les autorités de santé, les partenaires et les organismes communautaires provinciaux, régionaux et locaux. Les auteurs tiennent à remercier chaleureusement pour leur contribution les participants, les équipes des sites sentinelles (y compris les coordinateurs et les intervieweurs de l'enquête) et les investigateurs principaux de chaque site : M. Alary; K. Chokani; J. DeMille; B. Enns; M. Gully; B. Hanley; M. Hennink; P. Leclerc; C. Mackie; S. Marshall; C. Morissette; É. Roy; S. Shaw; C. Smith; A. Vanderlaan et $D$. Warren. Les auteurs tiennent également à remercier $\mathrm{C}$. Archibald pour ses conseils avant et pendant la collecte des données et pour son examen du manuscrit, ainsi que C. Daniuk pour son aide dans l'analyse des échantillons de sang séché.

C. Hui était responsable de l'échange de connaissances et de la mobilisation du réseau avec le Réseau ontarien de réduction des méfaits. Il est maintenant étudiant au doctorat (boursier Vanier des Instituts de recherche en santé du Canada) en études des politiques à l'Université Ryerson.

\section{Financement}

L'enquête Track auprès des utilisateurs de drogues injectables au Canada est financée par l'Agence de la santé publique du Canada, et bénéficie de contributions en nature de la part des autorités de santé et des organismes communautaires régionaux et locaux. 


\section{Références}

1. Statistique Canada. Peuples autochtones - Faits saillants en tableaux, Recensement de 2016. https://www12.statcan.gc.ca/ census-recensement/2016/dp-pd/hlt-fst/abo-aut/Tableau.cfm?Lang $=F r a \& T=101 \& S R=1 \& S=99 \& O=A \& R P P=25 \& P R=0 \& D 1=1 \& D 2=1 \& D 3$ $=1 \& T A B \mid D=2$

2. Agence de la santé publique du Canada. Résumé: Estimations de l'incidence et de la prévalence du $\mathrm{VIH}$, et des progrès réalisés par le Canada en ce qui concerne les cibles $90-90-90$ pour le $\mathrm{VIH}$ 2016. Ottawa (ON) : Gouvernement du Canada; 2018. https:// www.canada.ca/content/dam/phac-aspc/documents/services/ publications/diseases-conditions/summary-estimates-hiv-incidenc e-prevalence-canadas-progress-90-90-90/pub-fra.pdf

3. Services aux Autochtones Canada. L'Hépatite $C$ chez les Premières Nations vivant dans les réserves au Canada. SAC; 2016. https:// www.sac-isc.gc.ca/DAM/DAM-ISC-SAC/DAM-HLTH/STAGING/ texte-text/diseases-hepC_1569958386864_fra.pdf

4. Haddad N, Li JS, Totten S, McGuire M. Le VIH au Canada : Rapport de surveillance, 2017. Relevé des maladies transmissibles au Canada 2018;44(12):367-76. DO

5. World Health Organization/UNAIDS. Guidelines on surveillance among populations most at risk for HIV. Geneva (Switzerland): WHO; 2011. http://files.unaids.org/en/media/unaids/contentassets/ documents/epidemiology/2011/20110518_Surveillance_among_ most_at_risk.pdf

6. Tarasuk J, Zhang J, Lemyre A, Cholette F, Bryson M, Paquette D. Résultats nationaux de l'enquête Track auprès des utilisateurs de drogues injectables au Canada, phase 4, 2017 à 2019. Relevé des maladies transmissibles au Canada 2020;46(5):155-68. DOI
7. Bauer GR, Braimoh J, Scheim Al, Dharma C. Transgender-inclusive measures of sex/gender for population surveys: mixed-methods evaluation and recommendations. PLoS One 2017;12(5):e0178043. DOI PubMed

8. Services aux Autochtones Canada. Ligne d'écoute d'espoir. SAC; 2019. https://www.sac-isc.gc.ca/fra/1576089519527/1576089566478

9. Spittal PM, Pearce ME, Chavoshi N, Christian WM, Moniruzzaman A, Teegee M, Schechter MT. The Cedar Project: high incidence of HCV infections in a longitudinal study of young Aboriginal people who use drugs in two Canadian cities. BMC Public Health 2012;12:632. DOI PubMed

10. BC Centre for Disease Control. HIV in British Columbia: Annual Surveillance Report 2017. BCCDC; 2019. www.bccdc.ca/ health-professionals/data-reports/hiv-aids-reports

11. Craib KJ, Spittal PM, Wood E, Laliberte N, Hogg RS, Li K, Heath K, Tyndall MW, O'Shaughnessy MV, Schechter MT. Risk factors for elevated HIV incidence among Aboriginal injection drug users in Vancouver. CMAJ 2003;168(1):19-24. PubMed

12. Uhanova J, Tate RB, Tataryn DJ, Minuk GY. The epidemiology of hepatitis $C$ in a Canadian Indigenous population. Can J Gastroenterol 2013;27(6):336-40. DOI PubMed

13. Agence de la santé publique du Canada. Réduction des répercussions sur la santé des infections transmissibles sexuellement et par le sang au Canada d'ici 2030 : un cadre d'action pancanadien sur les ITSS. ASPC; 2018. https://www.canada.ca/ content/dam/phac-aspc/documents/services/infectious-diseases/ sexual-health-sexually-transmitted-infections/reports-publications/ sexually-transmitted-blood-borne-infections-action-framework/ infections-transmissibles-sexuellement-sang-cadre-action.pdf 


\section{Annexe 1 : Algorithmes de dépistage de l'immunodéficience humaine et de I'hépatite $C$}

\section{Algorithmes de dépistage du VIH}

Pour les sites extérieurs au réseau SurvUDI, le statut de positivité pour le $\mathrm{VIH}$ a dans un premier temps été déterminé en analysant les échantillons de sang séché au moyen de l'essai antigène-anticorps anti-VIH Bio-Rad GS, suivi de tests de confirmation réalisés à l'aide de l'essai quantitatif Roche COBAS AmpliPrep/COBAS Taqman VIH-1 v2.0 (London) ou de l'essai qualitatif Roche COBAS AmpliPrep/COBAS Taqman VIH-1 v2.0 (Nouveau-Brunswick, Terre-Neuve et Regina). Pour les autres sites extérieurs au réseau SurvUDI (île de Vancouver, Thunder Bay, Whitehorse, Winnipeg, Prince Albert et Hamilton), compte tenu de la récurrence des échantillons de faible volume, le statut de séropositivité pour le $\mathrm{VIH}$ a été déterminé par des tests de dépistage et des tests de confirmation basés sur deux essais immunoenzymatiques distincts. Ainsi, dans la plupart des cas, le volume des échantillons était suffisant pour vérifier la présence du $\mathrm{VIH}$ et de l'hépatite $\mathrm{C}$. II est peu probable que le changement apporté aux algorithmes ait pu influer sur les résultats. Les algorithmes sont décrits de manière plus détaillée ci-dessous.

London : Le dépistage du VIH a été réalisé au moyen de I'essai antigène-anticorps anti-VIH Bio-Rad GS. Un résultat non réactif témoignait d'une absence d'infection au $\mathrm{VIH}$. Des tests de confirmation ont été réalisés sur les résultats de dépistage réactifs au moyen de l'essai quantitatif Roche COBAS AmpliPrep/ COBAS Taqman VIH-1 v2.0. Un résultat détecté témoignait d'une infection au $\mathrm{VIH}$. Lorsque l'essai antigène-anticorps anti-VIH Bio-Rad GS était positif, et que le résultat de l'essai VIH-1 Roche COBAS ApliPrep/COBAS Taqman v2.0 n'était pas détecté, un second essai immunoenzymatique (système Microelisa AVIOQ $\mathrm{VIH}-1$ ) était réalisé. Un résultat réactif à l'essai antigène-anticorps anti-VIH Bio-Rad GS et au système Microelisa AVIOQ VIH-1 témoignait d'une infection au VIH.

Nouveau-Brunswick, Terre-Neuve et Regina : Le dépistage du $\mathrm{VIH}$ a été réalisé au moyen de l'essai antigène-anticorps anti-VIH Bio-Rad GS (essai Bio-Rad). Un résultat non réactif témoignait d'une absence d'infection au VIH. Des tests de confirmation ont été réalisés sur les résultats d'analyse réactifs au moyen de l'essai qualitatif Roche COBAS AmpliPrep/COBAS Taqman $\mathrm{VIH}-1 \mathrm{v} 2.0$ (essai Roche). Un résultat détecté témoignait d'une infection au VIH. Lorsque l'essai Bio-Rad était réactif, et que le résultat de l'essai Roche n'était pas détecté, un second essai immunoenzymatique, le système Microelisa AVIOO VIH-1 (essai Avioq) était réalisé pour apporter une confirmation définitive. Un résultat réactif à l'essai Bio-Rad et à l'essai Avioq témoignait d'une infection au $\mathrm{VIH}$. Un résultat réactif à l'essai Bio-Rad, un résultat non détecté à l'essai Roche et un résultat non réactif ou indéterminé (i.e. un résultat d'absorbance proche de la valeur limite associée à un résultat réactif ou non réactif, sans toutefois être identique à celle-ci) à l'essai Avioq étaient globalement interprétés comme un résultat indéterminé.
Île de Vancouver, Thunder Bay, Whitehorse, Winnipeg, Prince Albert et Hamilton : Le dépistage du $\mathrm{VIH}$ a été réalisé au moyen de l'essai antigène-anticorps anti-VIH Bio-Rad GS (essai Bio-Rad). Un résultat non réactif témoignait d'une absence d'infection au $\mathrm{VIH}$. Des tests de confirmation ont été réalisés sur les résultats de dépistage réactifs au moyen d'un second essai immunoenzymatique, le système Microelisa AVIOQ VIH-1 (essai Avioq). Un résultat réactif témoignait d'une infection au VIH. Lorsque l'essai Bio-Rad était réactif ou que l'essai Avioq était non réactif ou indéterminé (i.e. avec un résultat d'absorbance proche de la valeur limite associée à un résultat réactif ou non réactif, sans toutefois être identique à celle-ci), l'essai qualitatif $\mathrm{VIH}-1$ Roche COBAS AmpliPrep/COBAS Taqman v2.0 (essai Roche) était réalisé pour apporter une confirmation définitive. Un résultat réactif à l'essai Bio-Rad et un résultat détecté à l'essai Roche témoignaient d'une infection au $\mathrm{VIH}$. Un résultat réactif à l'essai Bio-Rad, un résultat non réactif ou indéterminé à l'essai Avioq et un résultat non détecté à l'essai Roche étaient globalement interprétés comme un résultat indéterminé.

Pour les sites du réseau SurvUDI, les échantillons de salive ont été soumis à des analyses visant à détecter la présence du VIH au Laboratoire de santé publique du Québec et à l'Institut national de santé publique du Québec; ces analyses ont été réalisées au moyen de l'essai immunoenzymatique Bio-Rad GS PLUS O VIH-1/VIH-2, un essai diagnostique approuvé par Santé Canada et validé dans l'étude SurvUDI pour les échantillons de salive. Des tests de confirmation n'ont pas été réalisés sur les échantillons associés à plusieurs reprises à un résultat réactif. Un résultat positif témoignait d'une infection au $\mathrm{VIH}$.

\section{Algorithmes de dépistage de l'hépatite C Pour les sites extérieurs au réseau SurvUDI : le dépistage de l'hépatite $C$ a été réalisé au moyen de l'essai} immunoenzymatique Ortho ${ }^{\circledR}$ VHC version 3.0 (essai Ortho). Un résultat non réactif témoignait d'une absence d'antécédents d'infection à l'hépatite $C$. Un résultat réactif témoignait d'une exposition à vie à l'hépatite $\mathrm{C}$. Des tests de confirmation ont été réalisés sur les résultats de dépistage réactifs et indéterminés (i.e. les résultats d'absorbance proches de la valeur limite associée à un résultat réactif ou non réactif, sans toutefois être identique à celle-ci) au moyen de l'essai quantitatif Roche COBAS AmpliPrep/COBAS Taqman VHC v2.0 (essai Roche). Un résultat détecté témoignait d'une infection actuelle à l'hépatite $C$ et un résultat non détecté témoignait d'une exposition à vie à l'hépatite $C$. Pour les échantillons associés à un résultat indéterminé à l'essai Ortho, un résultat détecté à l'essai Roche témoignait d'une infection actuelle à l'hépatite $C$ et un résultat non détecté à l'essai Roche était interprété comme un résultat indéterminé. 
Pour les sites du réseau SurvUDI : le dépistage des anticorps anti-hépatite $C$ dans les échantillons de salive a été réalisé au moyen de l'essai immunoenzymatique Ortho ${ }^{\circledR}$ hépatite $C$ version 3.0 par les laboratoires de l'Institut national de santé publique du Québec. Les tests de confirmation n'ont pas été réalisés sur les échantillons associés à plusieurs reprises à un résultat positif. Un résultat positif témoignait d'une infection antérieure ou actuelle à l'hépatite $C$, sans faire la distinction entre une infection aiguë, chronique ou passée. La validation de ce test en vue d'une utilisation avec les échantillons de salive a été effectuée dans l'étude SurvUDI.

\section{Sensibilité et spécificité des essais de laboratoire}

D'après la documentation du fabricant et les données de validation interne, la spécificité de l' essai immunoenzymatique antigène-anticorps anti-VIH Bio-Rad GS, du système Microelisa Avioq VIH-1 et de l'essai qualitatif Roche COBAS AmpliPrep/ COBAS TaqMan VIH-1 v2.0 est égale ou supérieure à 99,9\% sur les échantillons de sang séché. De même, chaque essai affiche une sensibilité de $100 \%$, à l'exception de l'essai immunoenzymatique antigène-anticorps anti-VIH Bio-Rad GS qui présente une sensibilité de 96,6\%. Le seuil de quantification de I'essai quantitatif Roche COBAS/AmpliPrep TaqMan VIH-1 v2.0 sur les échantillons de sang séché est de 616 copies $/ \mathrm{ml}$.

D'après les données de validation interne, le système d'essai ORTHO ELISA VHC v3.0 présente une spécificité et une sensibilité de $100 \%$. Le seuil de quantification de l'essai Roche COBAS AmpliPrep/COBAS TaqMan VHC v2.0 est de $355 \mathrm{IU} / \mathrm{ml}$.

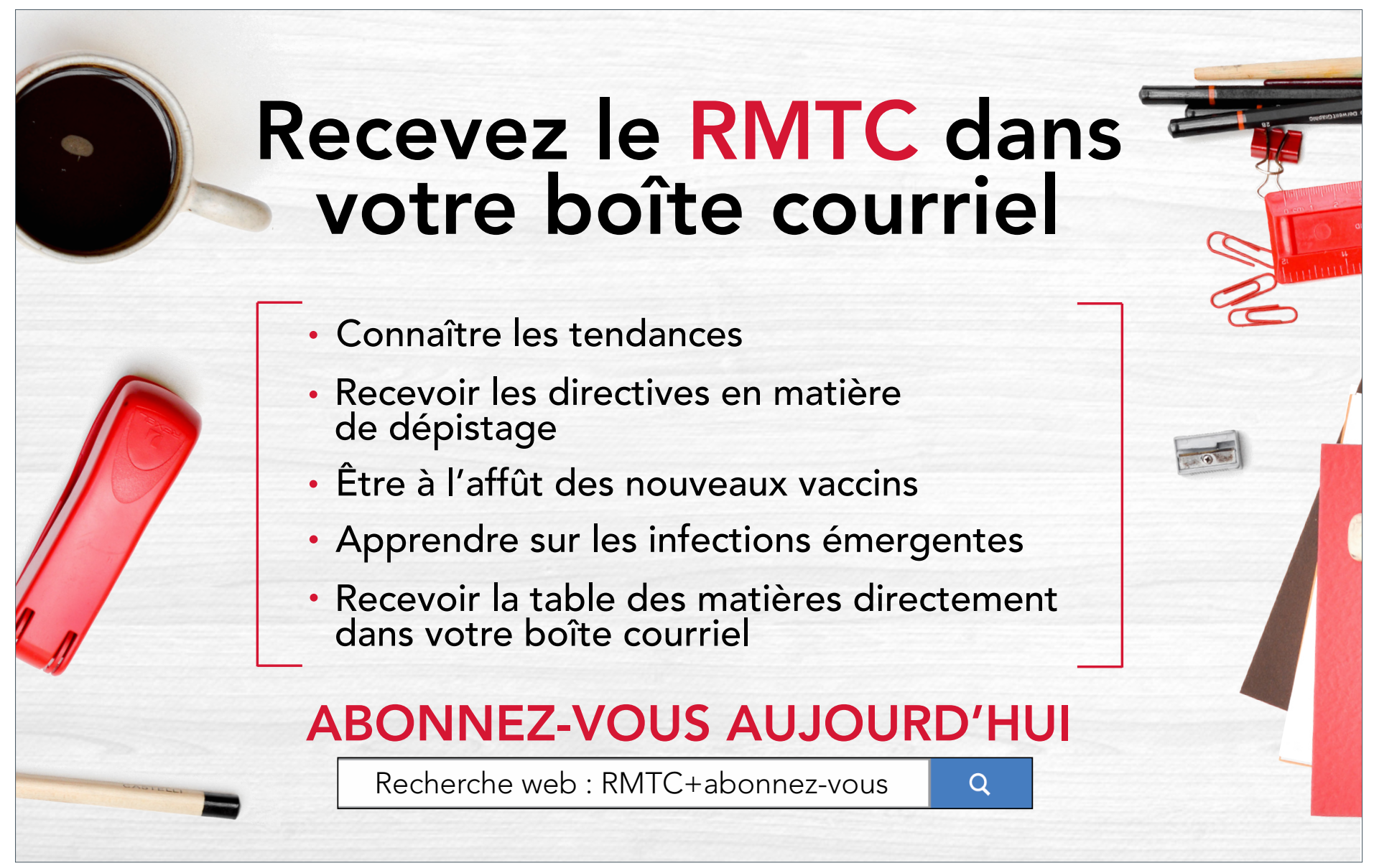

